\title{
Impact of nocturnal oxygen therapy for central sleep apnea syndrome on nocturnal premature ventricular contractions
}

We report here a case of central sleep apnea syndrome associated with frequent nocturnal premature ventricular contractions (PVCs) that were successfully eliminated using nocturnal oxygen therapy. He was admitted to our hospital for chest discomfort during the night. The symptoms frequently woke him up during sleep. His electrocardiogram (ECG) showed sinus rhythm accompanied by PVCs. The 24 hour ambulatory ECG detected frequent PVCs during the night (more than 1000 beats/hour). Additionally, polysomnography demonstrated severe central sleep apnea syndrome, the so-called Cheyne-Stokes respirations (apnea-hypopnea index 48/ hour). He received oxygen at a rate of 2 liter/min only during sleep. After nocturnal nasal oxygen therapy, the PVCs became significantly fewer and his symptoms disappeared. This case report suggests the possibility of an impact of oxygen inhalation during sleep in patients with central SAS on the prevention of ventricular arrhythmias during both the daytime and night. However, further well-established randomized, controlled studies are needed to confirm these effects.

Submitted: 20 September 2015; Accepted: 15 October 2015; Published online: 23 October 2015

Keywords: Sleep apnea - Premature ventricular contraction - Oxygen

The previous investigators reported the impact of obstructive sleep apnea syndrome (SAS) on atrial and ventricular arrhythmias. However, reports regarding central SAS are rare. We report here a case of central sleep apnea associated with frequent nocturnal PVCs that were successfully eliminated using only nocturnal nasal oxygen therapy.

\section{Case Report}

A 64-year-old man with chest discomfort during night was referred to our hospital. The symptoms occasionally interrupted his sleep. His body height and weight were $169 \mathrm{~cm}$ and $72 \mathrm{~kg}$. His body mass index (BMI) was $25.2 \mathrm{~kg} / \mathrm{m} 2$. He did not feel any severe palpitations or drowsiness during the daytime. No stertorous breathing was noticed by his family. His electrocardiogram (ECG) showed sinus rhythm (66/ $\mathrm{min}$ ) accompanied by premature ventricular contractions (PVCs). The origin of the PVCs was the right ventricular outflow tract (estimated by the morphology, Figure 1).

An echocardiogram showed a slightly diffuse reduction of the left ventricular contraction (ejection fraction, LVEF $55 \%$ ). Neither valvular disease nor any other abnormalities were identified. The plasma concentration of the brain natriuretic peptide (BNP) was almost within normal limits $(67 \mathrm{ng} / \mathrm{ml})$. The 24-hours ambulatory ECG monitoring demonstrated 12,521 PVCs out of a total
Takeshi Tomita1*, Takeo Higashikata', Ei-ichiro Mawatari', Tsunemi Koji', Harumi Sasaki², KojiYoshie ${ }^{3}$, Yasutaka Oguchi ${ }^{3}$ Takahiro Takeuchi Ayako Okada 3 Uichi Ikeda ${ }^{3}$ 'Department of Cardiovascular Medicine, North Alps Medical Center Azumi Hospital3207-1 Ikeda Kita-Azumi, Nagano, Japan

${ }^{2}$ Clinical laboratory, North Alps Medical Center Azumi Hospital 3207-1 Ikeda

Kita-Azumi, Nagano, Japan

${ }^{3}$ Department of Cardiovascular Medicine, Shinshu University School of Medicine3-1-1 Asahi Matsumoto, Nagano, Japan

*Author for correspondence: Tel.: +8126337348 ttomita@shinshu-u.ac.jp 


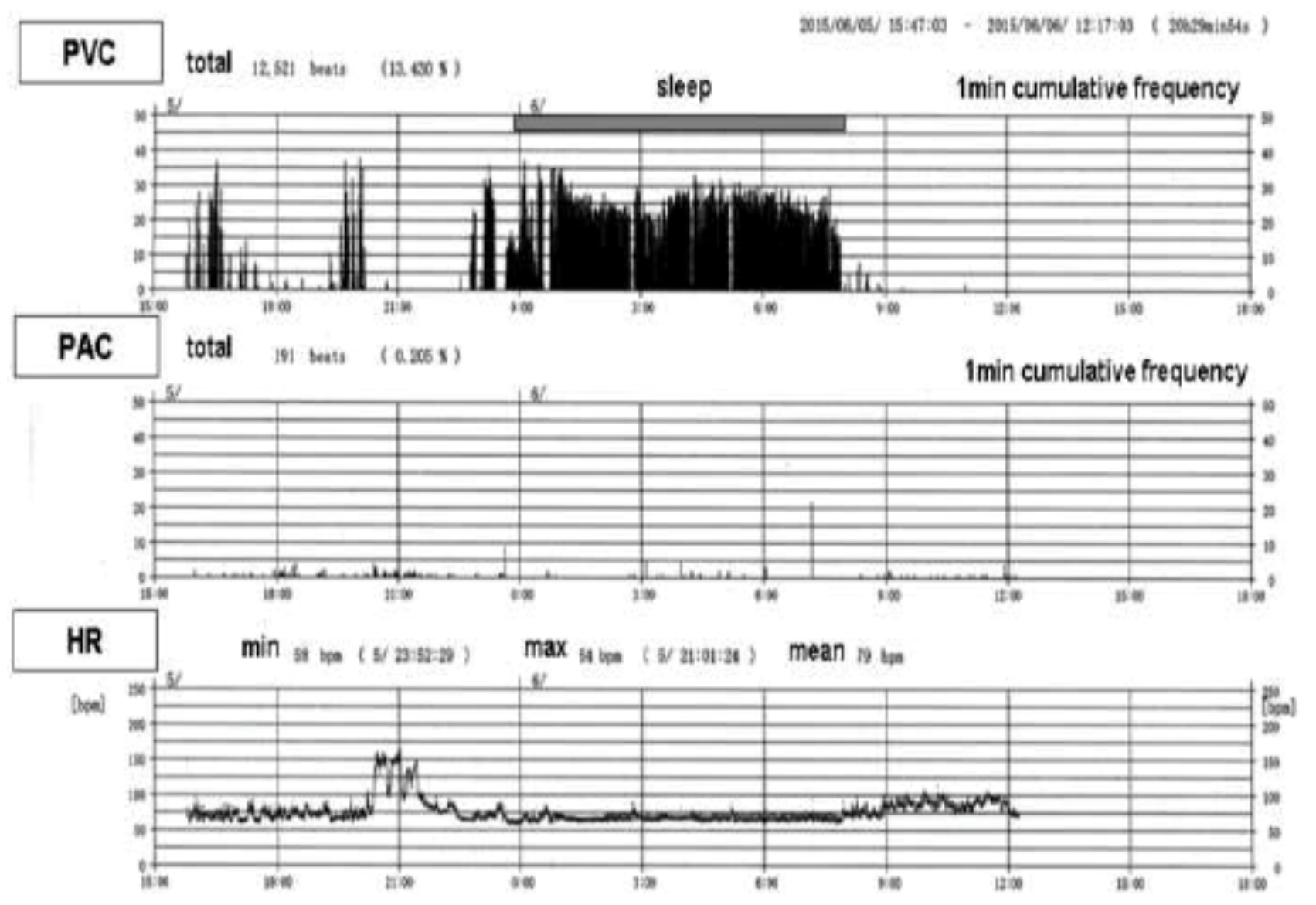

Figure 1: The 24-hour ambulatory ECG monitoring before oxygen therapy. The 24-hour ambulatory ECG monitoring on admission demonstrated 12,521 PVCs out of a total of 93,231 ventricular beats (13.4\%). The PVCs markedly increased, presenting as ventricular bigeminy, especially during sleep from 0:00 to 8:00 (more than 30\% of the total ventricular beats). PVC: Premature Ventricular Contraction; PAC: Premature Atrial Contraction; HR: Heart Rate.

of 93,231 ventricular beats (13.4\%). PVCs markedly increased presenting as ventricular bigeminy (more than $30 \%$ ) especially during sleep. The average heart rate during sleep was $68 / \mathrm{min}$. He had been treated with a beta-antagonist at another hospital. Unfortunately, that treatment was not effective and had already been discontinued. We performed polysomnography (PSG) to detect sleep disordered breathing (Figure 2).

The PSG demonstrated a pattern of severe central sleep apnea syndrome. The apnea and hypopnea index (AHI) was 47.9/hour (apnea index; 37.6 and hypopnea index; 10.3). The total duration of the Cheyne-Stokes respirations (CSRs) was 111 minutes $(27.0 \%$ of the total sleep time). The minimum SPO2 was $80 \%$ during the CSRs. The total sleep time was $412 \mathrm{~min}$. REM sleep, stage 1 , stage 2 , and stage 3 were $13.7 \%, 61.5 \%$, $14.3 \%$ and $10.5 \%$ of the total sleep time, respectively. The arousal index was 59.5/h. He received nasal oxygen at a rate of 2 liter/min only during sleep. We did not prescribe any anti-arrhythmic agents including beta- antagonists. After the nocturnal nasal oxygen therapy, the PVCs not only during sleep but also during the daytime significantly decreased and his nocturnal symptoms disappeared (Figure 3). The left ventricular contraction normalized (LVEF 62\%).

\section{Discussion}

Previous reports demonstrated that obstructive SAS was associated with ventricular arrhythmias and sudden cardiac death $[1,2]$. Since Sullivan et al. first reported continuous positive airway pressure therapy (CPAP) for the treatment of obstructive SAS it has become widespread as a treatment for obstructive SAS [3]. Several investigators have demonstrated the favorable effects of CPAP on cardiovascular events in patients with obstructive SAS [4]. On the other hand, previous available reports regarding the relationship between central SAS and ventricular arrhythmias are few. The present case had central sleep apnea accompanied by frequent nocturnal PVCs. Oxygen therapy during sleep 


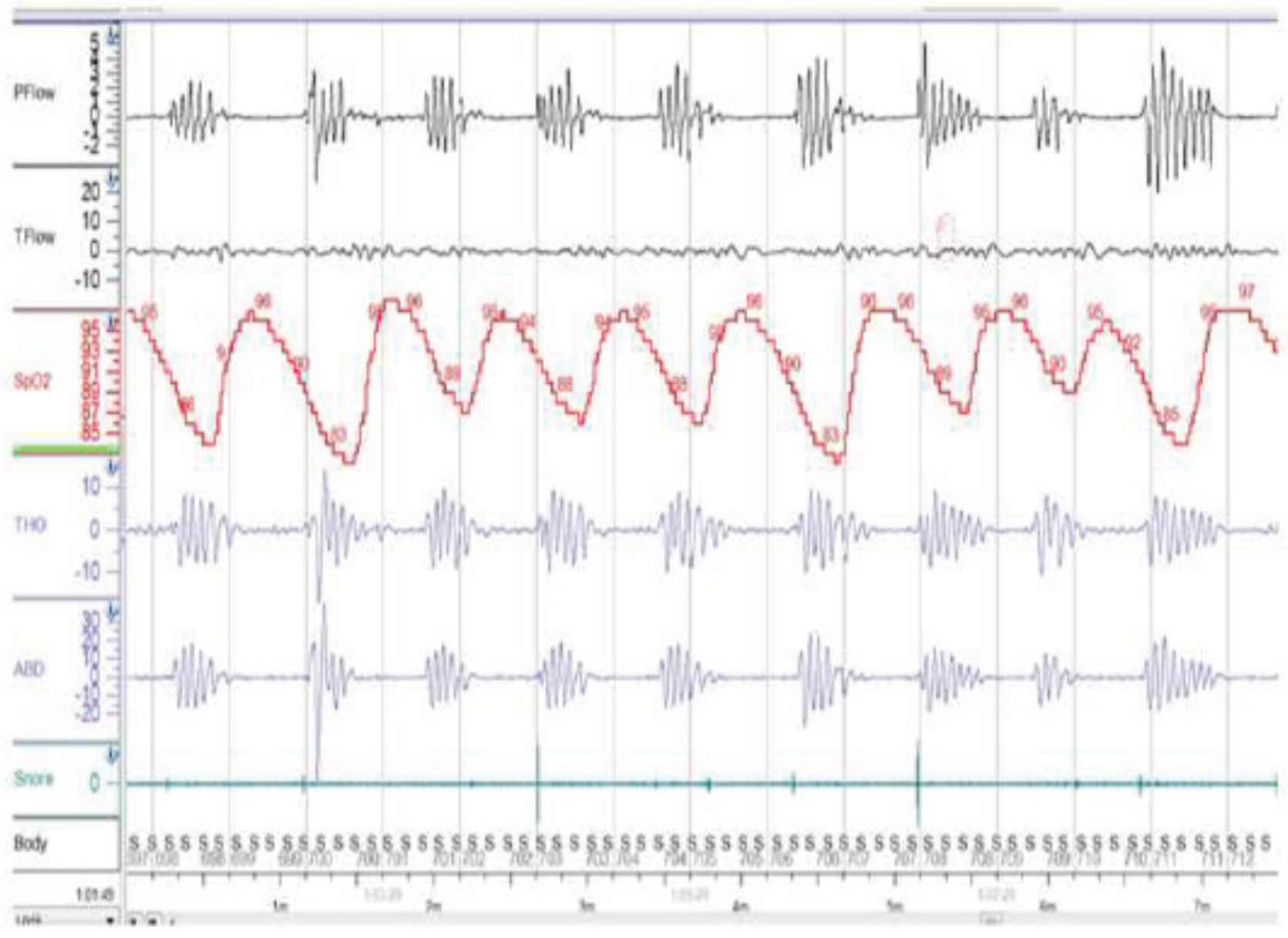

Figure 2: Polysomnography showed a pattern of central sleep apnea syndrome. The apnea and hypopnea index (AHI) was 47.9/hour. The total duration of Cheyne-Stokes respirations was 111 minute (27.0\% of the total sleep time). A representative example is noted in the panel. The Flow stopped periodically without any labored movement of the thorax or abdomen. PFlow: Pressure Flow; TFlow: Thermister Flow; THO: Thorax; ABO: Abdomen; S: Spine Position.

could successfully eliminate the PVCs not only during sleep but also during the daytime.

In generally, obstructive SAS results in recurrent hypoxemia and changes in the intracardiac pressure. These phenomena increase the sympathetic activity and potentially promote ventricular arrhythmias [5]. A CPAP prevents oxygen desaturation and changes in the intracardiac pressure, and reduce these adverse events [4]. However, controversy still exits about the effects of CPAP on ventricular arrhythmias and ventricular ectopic activity [6,7]. Treatment with nocturnal oxygen therapy for central SAS also improves the oxygen desaturation and prognosis in patients with heart failure [8]. Furthermore, an adequate treatment of SDB contributes to an improved sympathetic balance during the daytime as well as during the night, [8,9] and exercise capacity [10]. Recently, the SERVE-HF study showed that adaptive servo-ventilation therapy increases the all-cause and cardiovascular mortality in patients with symptomatic chronic heart failure and a reduced ejection fraction and predominant central sleep apnea [11]. In the present case without structural heart disease, nocturnal nasal oxygen inhalation could eliminate the PVCs during both the night and daytime without any anti-arrhythmic agents or catheter ablation. This case report suggests the possibility of an impact of oxygen inhalation during sleep in patients with central SAS on the prevention of ventricular arrhythmias during both the daytime and night. However, further well-established randomized, controlled studies are needed to confirm these effects.

\section{Conclusion}

We report a case with nocturnal frequent PVCs and central sleep apnea syndrome. PSG demonstrated severe central sleep apnea syndrome including Cheyne-Stokes respirations. After nocturnal nasal oxygen therapy, the PVCs not only during sleep but also during the daytime significantly decreased and his symptoms disappeared. 


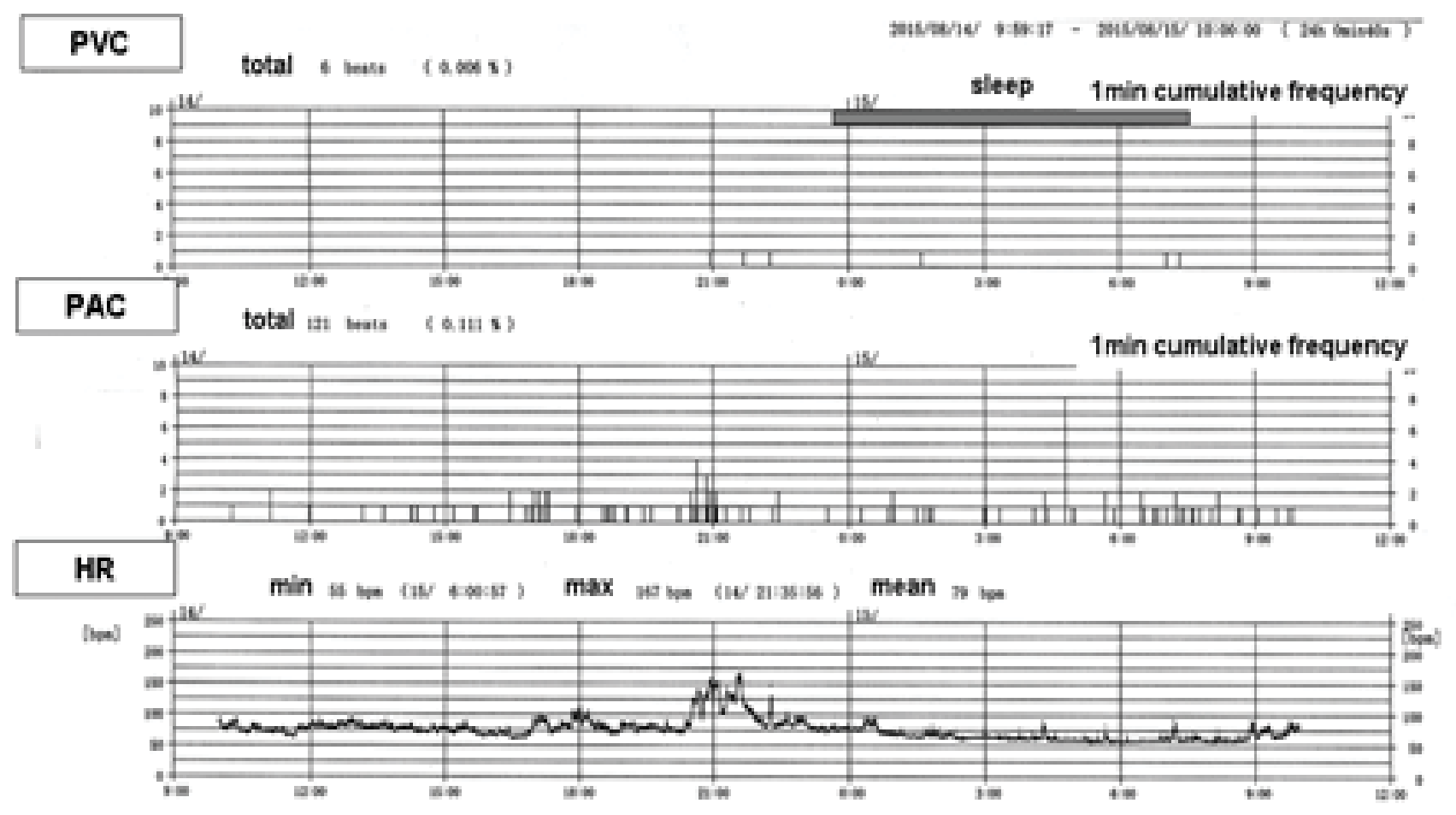

Figure 3: The 24-hours ambulatory ECG monitoring after the nocturnal nasal oxygen therapy. The 24-hours ambulatory ECG monitoring after the nocturnal nasal oxygen therapy at $2 \mathrm{~L} / \mathrm{min}$ with a nasal tube revealed the disappearance of the PVCs during both the daytime and night. PVC: Premature Ventricular Contraction; PAC: Premature Atrial Contraction; HR: Heart Rate.

\section{Executive summary}

- We report here a case of central sleep apnea syndrome associated with frequent nocturnal premature ventricular contractions (PVCs) that were successfully eliminated using nocturnal oxygen therapy. He was admitted to our hospital for chest discomfort during the night.

- This case report suggests the possibility of an impact of oxygen inhalation during sleep in patients with central SAS on the prevention of ventricular arrhythmias during both the daytime and night.

\section{References:}

1. Gami AS, Howard DE, Olson EJ, Somers VK. Day-night pattern of sudden death in obstructive sleep apnea. N. Engl. J. Med. 352, 1206-1214 (2005).

2. Gami AS, Olson EJ, Shen WK, et al. Obstructive sleep apnea and the risk of sudden cardiac death: a longitudinal study of 10,701 adults. J. Am. Coll. Cardiol. 62, 610-616 (2013).

3. Sullivan CE, Issa FG, Berthon-Jones M, Eves L. Reversal of obstructive sleep apnoea by continuous positive airway pressure applied through the nares. Lancet. 8225, 862-865 (1981).

4. McNicholas WT. Cardiovascular outcomes of CPAP therapy in obstructive sleep apnea syndrome. Am. J. Physiol. Regul. Integr. Comp. Physiol. 293: R1666-1670 (2007).

5. Mehra R, Benjamin EJ, Shahar E, et al. Association of nocturnal arrhythmias with sleep-disordered breathing: The Sleep Heart Health Study. Am. J. Respir. Crit. Care. Med. 173: 910-916 (2006).

6. Craig S, Pepperell JC, Kohler M, et al. Continuous positive airway pressure treatment for obstructive sleep apnoea reduces resting heart rate but does not affect dysrhythmias: a randomised controlled trial. J. Sleep. Res. 18: 329-336 (2009).
7. Roche F, Barthelemy JC, Garet M, et al. Continuous positive airway pressure treatment improves the QT rate dependence adaptation of obstructive sleep apnea patients. Pacing. Clin. Electrophysiol. 28, 819-825 (2005).

8. Sasayama S, Izumi T, Seino Y, Ueshima K, Asanoi H. Effects of nocturnal oxygen therapy on outcome measures in patients with chronic heart failure and cheyne-stokes respiration. Circ. J. 70, 1-7 (2006).

9. Staniforth AD, Kinnear WJ, Starling R, Hetmanski DJ, Cowley AJ. Effect of oxygen on sleep quality, cognitive function and sympathetic activity in patients with chronic heart failure and Cheyne-Stokes respiration. Eur. Heart. J. 19: 922-928 (1998).

10. Andreas S, Clemens C, Sandholzer H, Figulla HR, Kreuzer H. Improvement of exercise capacity with treatment of CheyneStokes respiration in patients with congestive heart failure. $J$. Am. Coll. Cardiol. 27, 1486-1490 (1996).

11. Cowie MR, Woehrle H, Wegscheider K, et al. Rationale and design of the SERVE-HF study: treatment of sleep- disordered breathing with predominant central sleep apnoea with adaptive servo-ventilation in patients with chronic heart failure. Eur. J. Heart. Fail. 15, 937-943 (2013). 